\title{
Genomic Copy Number Variation Affecting Genes Involved in the Cell Cycle Pathway: Implications for Somatic Mosaicism
}

\author{
Ivan Y. Iourov, ${ }^{1,2,3}$ Svetlana G. Vorsanova, ${ }^{1,2}$ Maria A. Zelenova, \\ Sergei A. Korostelev, ${ }^{4}$ and Yuri B. Yurov ${ }^{1,2}$ \\ ${ }^{1}$ Mental Health Research Center, Moscow 117152, Russia \\ ${ }^{2}$ Separated Structural Unit "Clinical Research Institute of Pediatrics", Pirogov Russian National Research Medical University, \\ Ministry of Health of Russian Federation, Moscow 125412, Russia \\ ${ }^{3}$ Department of Medical Genetics, Russian Medical Academy of Postgraduate Education, Moscow 123995, Russia \\ ${ }^{4}$ I.M. Sechenov First Moscow Medical University, Moscow 119991, Russia
}

Correspondence should be addressed to Ivan Y. Iourov; ivan.iourov@gmail.com

Received 11 March 2015; Accepted 27 July 2015

Academic Editor: Henry Heng

Copyright (c) 2015 Ivan Y. Iourov et al. This is an open access article distributed under the Creative Commons Attribution License, which permits unrestricted use, distribution, and reproduction in any medium, provided the original work is properly cited.

\begin{abstract}
Somatic genome variations (mosaicism) seem to represent a common mechanism for human intercellular/interindividual diversity in health and disease. However, origins and mechanisms of somatic mosaicism remain a matter of conjecture. Recently, it has been hypothesized that zygotic genomic variation naturally occurring in humans is likely to predispose to nonheritable genetic changes (aneuploidy) acquired during the lifetime through affecting cell cycle regulation, genome stability maintenance, and related pathways. Here, we have evaluated genomic copy number variation (CNV) in genes implicated in the cell cycle pathway (according to Kyoto Encyclopedia of Genes and Genomes/KEGG) within a cohort of patients with intellectual disability, autism, and/or epilepsy, in which the phenotype was not associated with genomic rearrangements altering this pathway. Benign CNVs affecting 20 genes of the cell cycle pathway were detected in 161 out of 255 patients (71.6\%). Among them, 62 individuals exhibited $>2$ CNVs affecting the cell cycle pathway. Taking into account the number of individuals demonstrating CNV of these genes, a support for this hypothesis appears to be presented. Accordingly, we speculate that further studies of CNV burden across the genes implicated in related pathways might clarify whether zygotic genomic variation generates somatic mosaicism in health and disease.
\end{abstract}

\section{Introduction}

Somatic mosaicism (somatic genome variations) has long been considered as a source for human genomic diversity and pathology [1-3]. However, causes and consequences of postzygotic genomic variation (i.e., loss/gain of chromosomes in a cell or aneuploidy) remain largely unknown. The latter is probably the reason for mosaicism underappreciation in current genomic research [2-4]. To date, somatic genome variations have been observed in almost all healthy human tissues [3-6]. Interestingly, somatic genetic changes more commonly manifest as aneuploidy [2-6]. Furthermore, it has been repeatedly shown that somatic aneuploidy is likely to be a mechanism for a variety of diseases [7-13]. Assessing causes and consequences of somatic genome variations, a hypothesis, suggesting genomic changes to be acquired during the lifetime because of natural zygotic genomic variation, has been proposed [14]. Since common types of somatic mosaicism (mainly postzygotic aneuploidy) are likely to result from alterations in cell division (mitotic) regulation and genome maintenance pathways $[4,13-15]$, it has been hypothesized that zygotic (heritable and sporadic) genomic variation across genes implicated in pathways related to cell cycle regulation is the most likely cause of intercellular genome diversification [14]. Consequently, a simple analysis of genomic copy number variation $(\mathrm{CNV})$ in genes implicated in these pathways is able to answer the question whether this hypothesis is worth further testing.

In the present study, we have performed an analysis of genomic CNV affecting genes implicated in the cell cycle 
pathway (hsa04110 from the Kyoto Encyclopedia of Genes and Genomes or KEGG) by high-resolution molecular karyotyping (SNP-microarray analysis) in a cohort of 225 children with intellectual disability, autism, epilepsy, and/or congenital malformations. Genomes of these individuals were addressed inasmuch as their phenotypes had resulted from genomic rearrangements (chromosome abnormalities), which had not affected genes implicated in this specific pathway.

\section{Materials and Methods}

2.1. Study Subjects. Genomes of 225 children with intellectual disability, autism, epilepsy, and/or congenital malformations from a cohort ( 2500 patients) that has been partially described in a previous study [16] were analyzed. These individuals were selected according to results of molecular karyotyping, which showed occurrence of genomic rearrangements (chromosome abnormalities) relevant to the phenotypes without affecting genes implicated in the cell cycle pathway (hsa04110 from KEGG). Patients' ages varied between 1 month and 18 years. Written informed parental consent was obtained for each individual.

2.2. CNV Analysis. Genomic CNVs were analyzed using CytoScan HD Arrays (Affymetrix, Santa Clara, CA) consisting of approximately 2.7 million markers for $\mathrm{CNV}$ evaluation and approximately 750,000 SNPs. CNVs were addressed by the Affymetrix Chromosome Analysis Suite (ChAS) software (ChAS analysis files for CytoScan HD Array version NA32.3). Genomic localization and gene content of detected CNVs were defined using NCBI Build GRCh37/hg19 reference sequence. The procedures have been previously described in detail [17-24].

2.3. Data Analysis. Data analysis was performed using a bioinformatic workflow described recently [25]. Data on individual CNV profiling was analyzed against all the genes indicated to be involved in the cell cycle pathway indexed in KEGG (http://www.genome.jp/dbget-bin/www_ bget?pathway+hsa04110). Inclusion criteria were referred to either a CNV affecting whole gene or an intragenic exonic copy number change. Causative CNVs (defined by a protocol of CNV prioritization [25]), submicroscopic genomic rearrangements, or larger chromosome abnormalities affecting these genes were all excluded from the analysis.

\section{Results and Discussion}

CNVs affecting genes implicated in the cell cycle pathway according to the KEGG (http://www.genome.jp/dbget-bin/ www_bget?pathway+hsa04110) were found in 161 patients (71.6\%). Twenty genes were affected in a variable manner (Figure 1). In total, $214 \mathrm{CNV}$ s have been detected. Recurrent CNVs affected SMC1A, RB1, CDC16, and CUL1 (deletions), STAG2 (duplications), and CDK6 and eighth exon of EP300 (four copies). It is to note that these genes were also affected by nonrecurrent CNVs. Deletions (one copy) have been observed in $70.6 \%$ (151 CNVs), duplications (three copies) in

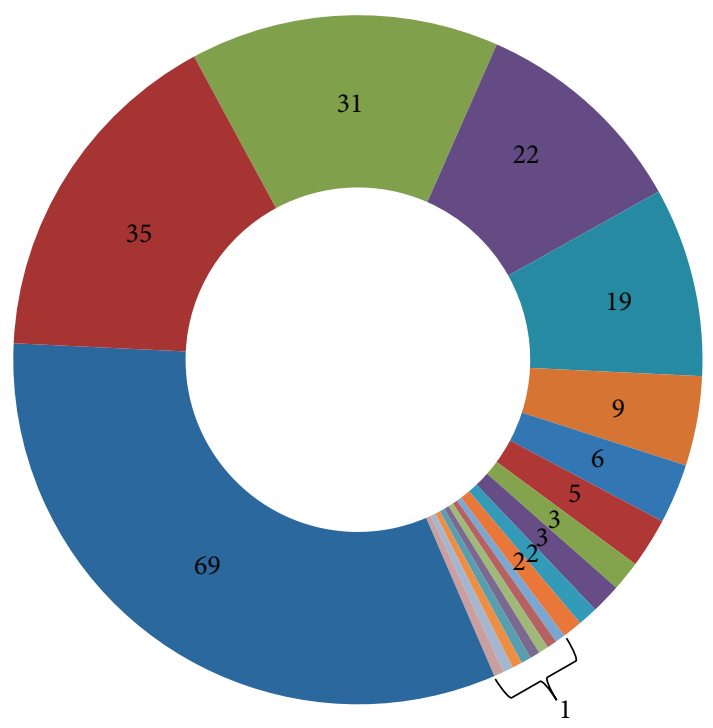

\begin{tabular}{l|l} 
SMC1A & CHEK2 \\
RB1 & STAG1 \\
EP300 & SMC1B \\
STAG2 & ANAPC10 \\
CDK6 & ESPL1 \\
CDC16 & CDC45 \\
CUL1 & CDKN1C \\
MAD1L1 & ABL1 \\
PCNA & RBL2 \\
TFDP1 & CCND2
\end{tabular}

FIGURE 1: Distribution of genomic CNVs (numbers correspond to amount of individuals demonstrating CNV affecting a gene) across genes implicated in the cell cycle pathway (hsa04110).

$16.8 \%$ (36 CNVs), and four copies in $12.6 \%$ (27 CNVs). In $M A D 1 L 1$, deletions and a copy number increase (four copies) were detected. In PCNA, CHEK2, STAG1, SMC1B, CDC45, and $A B L 1$ deletions were observed. In TFDP1, ESPL1, and $C D K N 1 C$ duplications were found. In ANAPC10, RBL2, and CCND2 other types of copy number increase (four copies) were detected.

Recurrent CNVs (apart from four copies of CDK6 and eighth exon of EP300) were all colocalized with genomic variations indexed in the Database of Genomic Variants of The Centre for Applied Genomics (TCAG) hosted databases at The Hospital for Sick Children (SickKids) (http://dgv.tcag.ca/dgv/app/home), whereas nonrecurrent $\mathrm{CNV}$ s were not found to correspond to genomic variations from the reference databases of benign genomic changes. These results suggest that a number of detected CNVs are common in general population.

Single CNVs affecting a gene implicated in the cell cycle pathway were found in 99 individuals. In the remainder, the incidence of the CNVs was as shown in Figure 2. Patterns of individual incidence of the CNV affecting genes implicated in the cell cycle pathway allowed us a suggestion that a kind 


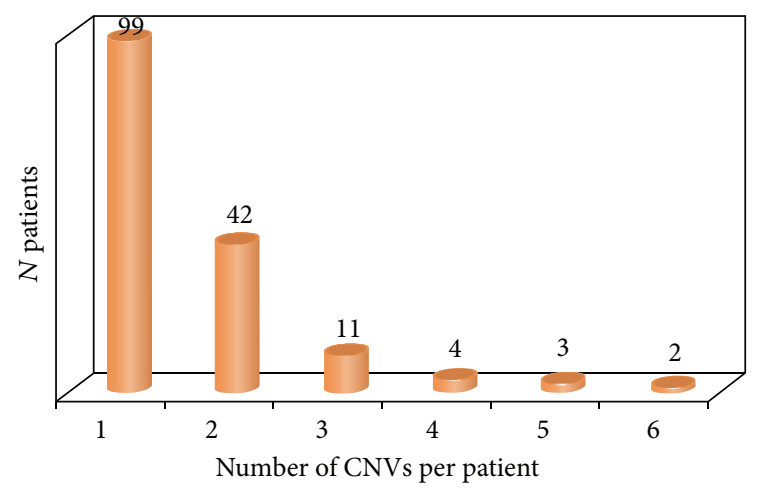

FIGURE 2: Individual incidence of CNV affecting genes implicated in the cell cycle pathway.

of CNV burden across the genes implicated in cell cycle pathways is likely to exist in at least $38.5 \%$ of individuals demonstrating genomic variations altering related pathways. Thus, discussions concerning a specific "cell cycle" CNV burden do not appear too speculative.

There is a line of evidence that somatic mosaicism is common in humans. Although somatic genome variations manifesting as structural chromosomal or genomic rearrangements are occasionally reported in unaffected population $[3,10,26-28]$, numerical chromosome abnormalities (aneuploidy and more rarely polyploidy including tissuespecific chromosomal mosaicism) [7, 12-14, 29-32] and small supernumerary marker chromosomes [33] are a common cause of somatic mosaicism. In addition, our cohort has been previously analyzed in terms of stochastic somatic chromosomal mosaicism and almost all individuals demonstrated low-level mosaic aneuploidy $[3,25,34,35]$. Moreover, human postmitotic tissues (i.e., adult human brain) demonstrate intercellular genomic variation essentially manifesting as low-level aneuploidy [32, 36-40]. Together, this suggests that a genomic background (i.e., CNV burden) for somatic genome diversification generated by alterations in cell cycle (genome stability) regulation pathways is likely to exist. The latter has been partially confirmed by studies of somatic genome variations mediating neurodegeneration resulting from alterations in cell cycle regulation and genome stability maintenance pathways [41-43]. Finally, numerous monogenic, chromosomal, and complex diseases are hypothesized to be associated with somatic mosaicism concomitant with failure of safeguarding genome and cell cycle machineries or genomic variations in related genes per se [44-51]. In the light of this study, it seems attractive to link presumably benign zygotic (sporadic or inherited) genomic variations slightly changing cell cycle pathway and somatic mosaicism. Alternatively, a heavier "cell cycle" CNV burden can be designated as a mechanism for a broad spectrum of diseases associated with somatic genome variations manifesting later in life [52, 53].

Somatic genome variations are considered to have prenatal origin. Developmental chromosome and genome instability hallmarks human prenatal development at cellular and tissular levels [54-60]. The following ontogenetic stages are also associated with changes of somatic cellular genomes. For instance, aging has long been documented to be associated with accumulation of sporadic somatic mutations, which were hypothesized to be produced either by exhaustion of mitotic and cell death machineries or by genomic variations affecting genes implicated in these pathways [38, 60-64]. Accordingly, ontogenetic genomic variation has been also attributed to these cellular pathways $[65,66]$. Similarly, addressing pathological aging of postmitotic tissues, it has been shown that these pathways are more likely to be inheritably altered rather than experience adverse changes during the lifespan [46, 67-69]. Nevertheless, environmental effects triggering accumulation of somatic mutations mediated by cell cycle errors represent an important contribution to healthy/unhealthy aging and a variety of aging and late onset diseases [70-74]. Consequently, our data supports the hypothesis about germline origins of genomic variations affecting genes implicated in cell cycle pathways that do predispose to somatic genome variations mediated by geneticenvironmental interactions. In this context, one can propose that a specific "cell cycle" CNV burden would be a key element in the pathogenic cascade initiated by constitutional (nonmosaic) genomic variation and culminated by somatic mosaicism.

$\mathrm{CNV}$ burden is a clinically valuable parameter that is important for assessing disease mechanisms and phenotypic significance of genomic variations [75-78]. However, this phenomenon has not been evaluated in cases of somatic mosaicism [25, 79]. An attempt at filling this gap by our preliminary data is pertinent inasmuch as the lack of an integral view on interaction between heritable/sporadic germline and somatic genome variations produces numerous discrepancies between empirical data acquired through singlecell analysis and generalized data on genome variability brought by "classical" strategies targeting DNA fractions isolated from large cell populations [79-81]. In this instance, mechanisms underlying intercellular genomic heterogeneity are likely to be referred to a predisposition of cellular genome to change. This suggests uncovering the basis of cellular genome susceptibility to vary throughout ontogeny to be of fundamental importance for current genomics and molecular genetic diagnosis.

Here, we have used KEGG for addressing contribution of CNVs to possible susceptibility to chromosome instability and to origin of somatic mosaicism. In silico analysis of CNV data has been considered contributive to definition of genetic mechanisms on the basis of molecular cytogenetic data [25]. Recently, KEGG-based selection/filtering of genes implicated in "pathways of interest" was found to be efficient for elucidating the molecular mechanisms of processes such as genome/chromosome instability and carcinogenesis involving genes found to be affected by CNVs in the present study [82-84]. Consequently, we concluded that gene ontology analysis of a single pathway in context of natural (presumably benign) CNVs is able to show whether further testing of the aforementioned hypothesis [52] would be productive.

The present data demonstrates that there do exist moreor-less common recurrent CNVs affecting 5 genes (SMC1A, RB1, EP300, STAG2, and CDK6) and rare but recurrent 
CNVs affecting 5 genes (CDC16, CUL1, MAD1L1, PCNA, and TFDP1) implicated in the cell cycle pathway (Figure 1). One can notice that detected CNVs are able to produce susceptibility to cancer mediated by chromosome/genome instability [85-87], which is rather predictable in the light of the involvement in the cell cycle pathway. In addition, a number of these genes are mutated in hereditary diseases. On the other hand, following guidelines on determination of $\mathrm{CNV}$ pathogenic value $[88,89]$ strongly evidences that these genomic changes are likely either to be benign or to produce a susceptibility to common diseases or traits. The latter can be considered mechanisms for increasing background levels of somatic (stochastic) mutations. Among genes implicated in the cell cycle pathway, SMC1A was most commonly involved in CNVs. This gene mutated in Cornelia de Lange syndrome and colorectal cancers [90] is involved in G2/M arrest in humans [91]. The second gene is $R B 1$ (retinoblastoma tumor-suppressor gene) representing a well-known inhibitor of cell cycle progression, alterations to which can cause aneuploidization and other processes initiating genome instability in cancers $[92,93]$. One can speculate that these recurrent CNVs are able to render cells susceptible to chromosome instability. EP300 is mutated in a small proportion of Rubinstein-Taybi syndrome cases [94] and in cancers exhibiting instable genomes, which can be a result of alterations to chromatin-remodeling [95]. Although inactivating point mutations in STAG2 are not likely to be directly related to aneuploidy [96], more recent studies have shown that frequent sequence variations are inversely related to chromosomal copy number changes [97]. CDK6 mutations causing clinical conditions and several cancers are involved in processes related to aneuploidization [98, 99]. Finally, CDC16, CUL1, MAD1L1, PCNA, and TFDP1 were all found to be integrated into a network of the cell cycle pathway, which is likely to be responsible for cancer progression [100] and involved in genome/chromosome instability. Thus, evaluating functional consequences of CNVs affecting the aforementioned genes is able to provide a basis for speculations concerning the ability of these apparently benign CNVs to be responsible for susceptibility to chromosome (genome) instability or somatic mosaicism in presumably normal tissues.

Our study provides a preliminary support for a hypothesis suggesting zygotic (sporadic and heritable) genomic variation to form a susceptibility to cellular genome instability or somatic genome variations (mosaicism) through genetic variability affecting genes implicated in cell cycle genome maintenance regulation pathways. Since this hypothesis appears to be valid at least in case of the cell cycle pathway (hsa04110), one may speculate that future studies targeted at evaluating related pathways (i.e., mitotic chromosome segregation, DNA reparation/replication, genome stability maintenance, etc.) are able to clarify whether zygotic genomic variation can generate somatic genome variation in health and disease.

\section{Conclusion}

Our preliminary study has shown that natural CNV affecting genes implicated in the cell cycle pathway is relatively common. It is noteworthy that a significant proportion of individuals with these CNVs carry a kind of CNV burden across genes implicated in the cell cycle pathway. These data provide an experimental support for the hypothesis suggesting natural zygotic genomic variation (heritable and sporadic) predisposing to nonheritable/postzygotic genomic changes (aneuploidy) affecting genes implicated in cell cycle regulation or related pathways acquired during the lifetime. Since an analysis of a single pathway, alterations in which result in somatic mosaicism (aneuploidy), could support the hypothesis, one may assume that increasing the numbers of pathways analyzed in this context would certainly give further insights into origins of somatic mosaicism and determine intrinsic interactions between zygotic and postzygotic genome variation.

\section{Consent}

Written consent was obtained from patients for publishing the results of this study.

\section{Conflict of Interests}

The authors declare no conflict of interests.

\section{Acknowledgments}

The paper is dedicated to Ilya V. Soloviev. The study was supported by the Russian Science Foundation (Project no.1415-00411).

\section{References}

[1] J. G. Hall, "Review and hypotheses: somatic mosaicism: observations related to clinical genetics," American Journal of Human Genetics, vol. 43, no. 4, pp. 355-363, 1988.

[2] A. J. Notini, J. M. Craig, and S. J. White, "Copy number variation and mosaicism," Cytogenetic and Genome Research, vol. 123, no. 1-4, pp. 270-277, 2008.

[3] I. Y. Iourov, S. G. Vorsanova, and Y. B. Yurov, "Chromosomal mosaicism goes global," Molecular Cytogenetics, vol. 1, article 26, 2008.

[4] A. Piotrowski, C. E. G. Bruder, R. Andersson et al., "Somatic mosaicism for copy number variation in differentiated human tissues," Human Mutation, vol. 29, no. 9, pp. 1118-1124, 2008.

[5] T. Wada and F. Candotti, "Somatic mosaicism in primary immune deficiencies," Current Opinion in Allergy \& Clinical Immunology, vol. 8, no. 6, pp. 510-514, 2008.

[6] N. G. Coufal, J. L. Garcia-Perez, G. E. Peng et al., "L1 retrotransposition in human neural progenitor cells," Nature, vol. 460, no. 7259, pp. 1127-1131, 2009.

[7] I. Y. Iourov, S. G. Vorsanova, and Y. B. Yurov, "Somatic genome variations in health and disease," Current Genomics, vol. 11, no. 6, pp. 387-396, 2010.

[8] H. H. Q. Heng, "Missing heritability and stochastic genome alterations," Nature Reviews Genetics, vol. 11, no. 11, p. 813, 2010.

[9] D. Freed, E. Stevens, and J. Pevsner, "Somatic mosaicism in the human genome," Genes, vol. 5, no. 4, pp. 1064-1094, 2014. 
[10] B. Rodríguez-Santiago, N. Malats, N. Rothman et al., "Mosaic uniparental disomies and aneuploidies as large structural variants of the human genome," The American Journal of Human Genetics, vol. 87, no. 1, pp. 129-138, 2010.

[11] S. De, "Somatic mosaicism in healthy human tissues," Trends in Genetics, vol. 27, no. 6, pp. 217-223, 2011.

[12] I. Y. Iourov, S. G. Vorsanova, and Y. B. Yurov, "Chromosomal variation in mammalian neuronal cells: known facts and attractive hypotheses," International Review of Cytology, vol. 249, pp. 143-191, 2006.

[13] R. P. Erickson, "Somatic gene mutation and human disease other than cancer: an update," Mutation Research/Reviews in Mutation Research, vol. 705, no. 2, pp. 96-106, 2010.

[14] J. P. Dumanski and A. Piotrowski, "Structural genetic variation in the context of somatic mosaicism," Methods in Molecular Biology, vol. 838, pp. 249-272, 2012.

[15] T. Liehr, E. Klein, K. Mrasek et al., "Clinical impact of somatic mosaicism in cases with small supernumerary marker chromosomes," Cytogenetic and Genome Research, vol. 139, no. 3, pp. 158-163, 2013.

[16] I. Y. Iourov, S. G. Vorsanova, O. S. Kurinnaia, M. A. Zelenova, A. P. Silvanovich, and Y. B. Yurov, "Molecular karyotyping by array CGH in a Russian cohort of children with intellectual disability, autism, epilepsy and congenital anomalies," Molecular Cytogenetics, vol. 5, article 46, 2012.

[17] C. Robberecht, T. Voet, G. E. Utine et al., "Meiotic errors followed by two parallel postzygotic trisomy rescue events are a frequent cause of constitutional segmental mosaicism," Molecular Cytogenetics, vol. 5, article 19, 2012.

[18] H. Mason-Suares, W. Kim, L. Grimmett et al., "Density matters: comparison of array platforms for detection of copy-number variation and copy-neutral abnormalities," Genetics in Medicine, vol. 15, no. 9, pp. 706-712, 2013.

[19] I. Y. Iourov, S. G. Vorsanova, V. Y. Voinova et al., "Xq28 (MECP2) microdeletions are common in mutation-negative females with Rett syndrome and cause mild subtypes of the disease," Molecular Cytogenetics, vol. 6, no. 1, article 53, 2013.

[20] Y. P. Yu, A. Michalopoulos, Y. Ding, G. Tseng, and J.-H. Luo, "High fidelity copy number analysis of formalin-fixed and paraffin-embedded tissues using Affymetrix Cytoscan HD chip," PLoS ONE, vol. 9, no. 4, Article ID e92820, 2014.

[21] C. Holzmann, D. N. Markowski, D. Koczan, B. M. Helmke, and J. Bullerdiek, "Genome-wide acquired uniparental disomy as well as chromosomal gains and losses in an uterine epithelioid leiomyoma," Molecular Cytogenetics, vol. 7, article 19, 2014.

[22] C. Liao, R. Li, F. Fu et al., "Prenatal diagnosis of congenital heart defect by genome-wide high-resolution SNP array," Prenatal Diagnosis, vol. 34, no. 9, pp. 858-863, 2014.

[23] B. T. Wang, T. P. Chong, F. Z. Boyar et al., "Abnormalities in spontaneous abortions detected by G-banding and chromosomal microarray analysis (CMA) at a national reference laboratory," Molecular Cytogenetics, vol. 7, article 33, 2014.

[24] F. R. Zahir and M. A. Marra, "Use of affymetrix arrays in the diagnosis of gene copy-number variation," Current Protocols in Human Genetics, vol. 85, pp. 8.13.1-8.13.13, 2015.

[25] I. Y. Iourov, S. G. Vorsanova, and Y. B. Yurov, "In silico molecular cytogenetics: a bioinformatic approach to prioritization of candidate genes and copy number variations for basic and clinical genome research," Molecular Cytogenetics, vol. 7, article 98, 2014.
[26] P. A. Jacobs, "Mutation rates of structural chromosome rearrangements in man," The American Journal of Human Genetics, vol. 33, no. 1, pp. 44-54, 1981.

[27] A. Kleczkowska, J. P. Fryns, and H. Van den Berghe, "On the variable effect of mosaic normal/balanced chromosomal rearrangements in man," Journal of Medical Genetics, vol. 27, no. 8, pp. 505-507, 1990.

[28] L. Y. F. Hsu, "Phenotype/karyotype correlations of Y chromosome aneuploidy with emphasis on structural aberrations in postnatally diagnosed cases," American Journal of Medical Genetics, vol. 53, no. 2, pp. 108-140, 1994.

[29] J. B. Geigl, S. Langer, S. Barwisch, K. Pfleghaar, G. Lederer, and M. R. Speicher, "Analysis of gene expression patterns and chromosomal changes associated with aging," Cancer Research, vol. 64, no. 23, pp. 8550-8557, 2004.

[30] B. Gottlieb, L. K. Beitel, and M. A. Trifiro, "Somatic mosaicism and variable expressivity," Trends in Genetics, vol. 17, no. 2, pp. 79-82, 2001.

[31] H. Youssoufian and R. E. Pyeritz, "Mechanisms and consequences of somatic mosaicism in humans," Nature Reviews Genetics, vol. 3, no. 10, pp. 748-758, 2002.

[32] I. Y. Iourov, T. Liehr, S. G. Vorsanova, A. D. Kolotii, and Y. B. Yurov, "Visualization of interphase chromosomes in postmitotic cells of the human brain by multicolour banding (MCB)," Chromosome Research, vol. 14, no. 3, pp. 223-229, 2006.

[33] T. Liehr, K. Mrasek, A. Weise et al., "Small supernumerary marker chromosomes-progress towards a genotypephenotype correlation," Cytogenetic and Genome Research, vol. 112, no. 1-2, pp. 23-34, 2006.

[34] Y. B. Yurov, S. G. Vorsanova, I. Y. Iourov et al., "Unexplained autism is frequently associated with low-level mosaic aneuploidy," Journal of Medical Genetics, vol. 44, no. 8, pp. 521-525, 2007.

[35] M. A. Hultén, J. Jonasson, E. Iwarsson et al., "Trisomy 21 mosaicism: we may all have a touch of down syndrome," Cytogenetic and Genome Research, vol. 139, no. 3, pp. 189-192, 2013.

[36] I. Y. Iourov, S. G. Vorsanova, T. Liehr, and Y. B. Yurov, "Aneuploidy in the normal, Alzheimer's disease and ataxiatelangiectasia brain: differential expression and pathological meaning," Neurobiology of Disease, vol. 34, no. 2, pp. 212-220, 2009.

[37] S. K. Rehen, Y. C. Yung, M. P. McCreight et al., "Constitutional aneuploidy in the normal human brain," Journal of Neuroscience, vol. 25, no. 9, pp. 2176-2180, 2005.

[38] Y. B. Yurov, I. Y. Iourov, V. V. Monakhov, I. V. Soloviev, V. M. Vostrikov, and S. G. Versanova, "The variation of aneuploidy frequency in the developing and adult human brain revealed by an interphase FISH study," Journal of Histochemistry \& Cytochemistry, vol. 53, no. 3, pp. 385-390, 2005.

[39] J. W. Westra, S. E. Peterson, Y. C. Yung, T. Mutoh, S. Barral, and J. Chun, "Aneuploid mosaicism in the developing and adult cerebellar cortex," Journal of Comparative Neurology, vol. 507, no. 6, pp. 1944-1951, 2008.

[40] J. W. Westra, R. R. Rivera, D. M. Bushman et al., "Neuronal DNA content variation (DCV) with regional and individual differences in the human brain," Journal of Comparative Neurology, vol. 518, no. 19, pp. 3981-4000, 2010.

[41] I. Y. Iourov, S. G. Vorsanova, T. Liehr, A. D. Kolotii, and Y. B. Yurov, "Increased chromosome instability dramatically disrupts neural genome integrity and mediates cerebellar degeneration 
in the ataxia-telangiectasia brain," Human Molecular Genetics, vol. 18, no. 14, pp. 2656-2669, 2009.

[42] T. Arendt, M. K. Brückner, B. Mosch, and A. Lösche, "Selective cell death of hyperploid neurons in Alzheimer's disease," American Journal of Pathology, vol. 177, no. 1, pp. 15-20, 2010.

[43] D. K. Jeppesen, V. A. Bohr, and T. Stevnsner, "DNA repair deficiency in neurodegeneration," Progress in Neurobiology, vol. 94, no. 2, pp. 166-200, 2011.

[44] R. D. Kennedy and A. D. D’Andrea, "DNA repair pathways in clinical practice: lessons from pediatric cancer susceptibility syndromes," Journal of Clinical Oncology, vol. 24, no. 23, pp. 3799-3808, 2006.

[45] R. Hochstenbach, J. E. Buizer-Voskamp, J. A. S. Vorstman, and R. A. Ophoff, "Genome arrays for the detection of copy number variations in idiopathic mental retardation, idiopathic generalized epilepsy and neuropsychiatric disorders: lessons for diagnostic workflow and research," Cytogenetic and Genome Research, vol. 135, no. 3-4, pp. 174-202, 2011.

[46] I. Y. Iourov, S. G. Vorsanova, and Y. B. Yurov, "Single cell genomics of the brain: focus on neuronal diversity and neuropsychiatric diseases," Current Genomics, vol. 13, no. 6, pp. 477488, 2012.

[47] C. Clauson, O. D. Schärer, and L. Niedernhofer, "Advances in understanding the complex mechanisms of DNA inter strand cross-link repair," Cold Spring Harbor Perspectives in Medicine, vol. 3, no. 10, Article ID a012732, 2013.

[48] G. Rossi, D. Conconi, E. Panzeri et al., "Mutations in MAPT gene cause chromosome instability and introduce copy number variations widely in the genome," Journal of Alzheimer's Disease, vol. 33, no. 4, pp. 969-982, 2013.

[49] Y.-C. Tang and A. Amon, "Gene copy-number alterations: a cost-benefit analysis," Cell, vol. 152, no. 3, pp. 394-405, 2013.

[50] G. Rossi, D. Conconi, E. Panzeri et al., "Mutations in MAPT give rise to aneuploidy in animal models of tauopathy," Neurogenetics, vol. 15, no. 1, pp. 31-40, 2014.

[51] L. A. Forsberg, D. Absher, and J. P. Dumanski, "Non-heritable genetics of human disease: spotlight on post-zygotic genetic variation acquired during lifetime," Journal of Medical Genetics, vol. 50, no. 1, pp. 1-10, 2013.

[52] I. Y. Iourov, S. G. Vorsanova, and Y. B. Yurov, "Somatic cell genomics of brain disorders: a new opportunity to clarify genetic-environmental interactions," Cytogenetic and Genome Research, vol. 139, no. 3, pp. 181-188, 2013.

[53] G. Desachy, L. A. Croen, A. R. Torres et al., "Increased female autosomal burden of rare copy number variants in human populations and in autism families," Molecular Psychiatry, vol. 20, no. 2, pp. 170-175, 2015.

[54] Y. B. Yurov, I. Y. Iourov, S. G. Vorsanova et al., "Aneuploidy and confined chromosomal mosaicism in the developing human brain," PLoS ONE, vol. 2, no. 6, article e558, 2007.

[55] M. A. Kingsbury, Y. C. Yung, S. E. Peterson, J. W. Westra, and J. Chun, "Aneuploidy in the normal and diseased brain," Cellular and Molecular Life Sciences, vol. 63, no. 22, pp. 2626-2641, 2006.

[56] J. F. Weier, H.-U. G. Weier, C. J. Jung et al., "Human cytotrophoblasts acquire aneuploidies as they differentiate to an invasive phenotype," Developmental Biology, vol. 279, no. 2, pp. 420432, 2005.

[57] Y. B. Yurov, S. G. Vorsanova, and I. Y. Iourov, “GIN'n'CIN hypothesis of brain aging: deciphering the role of somatic genetic instabilities and neural aneuploidy during ontogeny," Molecular Cytogenetics, vol. 2, article 23, 2009.
[58] H. Mkrtchyan, M. Gross, S. Hinreiner et al., "Early embryonic chromosome instability results in stable mosaic pattern in human tissues," PLoS ONE, vol. 5, no. 3, Article ID e9591, 2010.

[59] C. Robberecht, E. Vanneste, A. Pexsters, T. D’Hooghe, T. Voet, and J. R. Vermeesch, "Somatic genomic variations in early human prenatal development," Current Genomics, vol. 11, no. 6, pp. 397-401, 2010.

[60] Y. B. Yurov, S. G. Vorsanova, and I. Y. Iourov, "Ontogenetic variation of the human genome," Current Genomics, vol. 11, no. 6, pp. 420-425, 2010.

[61] F. Faggioli, T. Wang, J. Vijg, and C. Montagna, "Chromosomespecific accumulation of aneuploidy in the aging mouse brain," Human Molecular Genetics, vol. 21, no. 24, Article ID dds375, pp. 5246-5253, 2012.

[62] H.-G. Fischer, M. Morawski, M. K. Brückner, A. Mittag, A. Tarnok, and T. Arendt, "Changes in neuronal DNA content variation in the human brain during aging," Aging Cell, vol. 11, no. 4, pp. 628-633, 2012.

[63] R. M. Ricke and J. M. van Deursen, "Aneuploidy in health, disease, and aging," Journal of Cell Biology, vol. 201, no. 1, pp. 11-21, 2013.

[64] A. Behrens, J. M. van Deursen, K. L. Rudolph, and B. Schumacher, "Impact of genomic damage and ageing on stem cell function," Nature Cell Biology, vol. 16, no. 3, pp. 201-207, 2014.

[65] Y. B. Yurov, S. G. Vorsanova, and I. Y. Iourov, "The DNA replication stress hypothesis of Alzheimer's disease," TheScientificWorldJournal, vol. 11, pp. 2602-2612, 2011.

[66] F. Coppedè and L. Migliore, "Dna repair in premature aging disorders and neurodegeneration," Current Aging Science, vol. 3, no. 1, pp. 3-19, 2010.

[67] A. Bartke, "Single-gene mutations and healthy ageing in mammals," Philosophical Transactions of the Royal Society B: Biological Sciences, vol. 366, no. 1561, pp. 28-34, 2011.

[68] L. Migliore, F. Coppedè, M. Fenech, and P. Thomas, "Association of micronucleus frequency with neurodegenerative diseases," Mutagenesis, vol. 26, no. 1, pp. 85-92, 2011.

[69] F. Coppedè and L. Migliore, "DNA damage in neurodegenerative diseases," Mutation Research/Fundamental and Molecular Mechanisms of Mutagenesis, vol. 776, pp. 84-97, 2015.

[70] A. Granic and H. Potter, "Mitotic spindle defects and chromosome mis-segregation induced by LDL/cholesterolimplications for Niemann-Pick C1, Alzheimer's disease, and atherosclerosis," PLoS ONE, vol. 8, no. 4, Article ID e60718, 2013.

[71] P. Katsel, W. Tan, P. Fam, D. P. Purohit, and V. Haroutunian, "Cell cycle checkpoint abnormalities during dementia: a plausible association with the loss of protection against oxidative stress in Alzheimer's disease," PLoS ONE, vol. 8, no. 7, Article ID e68361, 2013.

[72] K. A. Ross, "Coherent somatic mutation in autoimmune disease," PLoS ONE, vol. 9, no. 7, Article ID e101093, 2014.

[73] Y. Yang, C. Shepherd, and G. Halliday, "Aneuploidy in Lewy body diseases," Neurobiology of Aging, vol. 36, no. 3, pp. 12531260, 2015.

[74] Y. B. Yurov, S. G. Vorsanova, T. Liehr, A. D. Kolotii, and I. Y. Iourov, "X chromosome aneuploidy in the Alzheimer's disease brain," Molecular Cytogenetics, vol. 7, no. 1, article 20, 2014.

[75] C. Lee and S. W. Scherer, "The clinical context of copy number variation in the human genome," Expert Reviews in Molecular Medicine, vol. 12, article e8, 2010. 
[76] S. G. Vorsanova, Y. B. Yurov, I. V. Soloviev, and I. Y. Iourov, "Molecular cytogenetic diagnosis and somatic genome variations," Current Genomics, vol. 11, no. 6, pp. 440-446, 2010.

[77] S. Girirajan and E. E. Eichler, "Phenotypic variability and genetic susceptibility to genomic disorders," Human Molecular Genetics, vol. 19, no. 2, pp. R176-R187, 2010.

[78] Y. Bromberg, "Building a genome analysis pipeline to predict disease risk and prevent disease," Journal of Molecular Biology, vol. 425, no. 21, pp. 3993-4005, 2013.

[79] I. Y. Iourov, S. G. Vorsanova, and Y. B. Yurov, "Intercellular genomic (chromosomal) variations resulting in somatic mosaicism: mechanisms and consequences," Current Genomics, vol. 7, no. 7, pp. 435-446, 2006.

[80] B. Y. Abdallah, S. D. Horne, J. B. Stevens et al., "Single cell heterogeneity: why unstable genomes are incompatible with average profiles," Cell Cycle, vol. 12, no. 23, pp. 3640-3649, 2013.

[81] K. A. Knouse, J. Wu, C. A. Whittaker, and A. Amon, "Single cell sequencing reveals low levels of aneuploidy across mammalian tissues," Proceedings of the National Academy of Sciences of the United States of America, vol. 111, no. 37, pp. 13409-13414, 2014.

[82] Z. Li, B.-Q. Li, M. Jiang et al., "Prediction and analysis of retinoblastoma related genes through gene ontology and KEGG," BioMed Research International, vol. 2013, Article ID 304029, 8 pages, 2013

[83] J. Yang, L. Chen, X. Kong, T. Huang, and Y. D. Cai, "Analysis of tumor suppressor genes based on gene ontology and the KEGG pathway," PLoS ONE, vol. 9, no. 9, Article ID e107202, 2014.

[84] C. Xing, R. Zhang, J. Cui et al., "Pathway crosstalk analysis of non-small cell lung cancer based on microarray gene expression profiling," Tumori Journal, vol. 101, no. 1, pp. 111-116, 2015.

[85] B. Vogelstein and K. W. Kinzler, "Cancer genes and the pathways they control," Nature Medicine, vol. 10, no. 8, pp. 789-799, 2004.

[86] A. Miremadi, M. Z. Oestergaard, P. D. P. Pharoah, and C. Caldas, "Cancer genetics of epigenetic genes," Human Molecular Genetics, vol. 16, no. 1, pp. R28-R49, 2007.

[87] M. S. Lawrence, P. Stojanov, C. H. Mermel et al., "Discovery and saturation analysis of cancer genes across 21 tumour types," Nature, vol. 505, no. 7484, pp. 495-501, 2014.

[88] S. W. Scherer, C. Lee, E. Birney et al., "Challenges and standards in integrating surveys of structural variation," Nature Genetics, vol. 39, no. 1, pp. S7-S15, 2007.

[89] J. R. Vermeesch, H. Fiegler, N. de Leeuw et al., "Guidelines for molecular karyotyping in constitutional genetic diagnosis," European Journal of Human Genetics, vol. 15, no. 11, pp. 11051114,2007

[90] L. Mannini, J. Liu, I. D. Krantz, and A. Musio, "Spectrum and consequences of SMC1A mutations: the unexpected involvement of a core component of cohesin in human disease," Human Mutation, vol. 31, no. 1, pp. 5-10, 2010.

[91] Z. Ma, M. Lin, K. Li et al., "Knocking down SMC1A inhibits growth and leads to G2/M arrest in human glioma cells," International Journal of Clinical and Experimental Pathology, vol. 6, no. 5, pp. 862-869, 2013.

[92] D. W. Goodrich, "The retinoblastoma tumor-suppressor gene, the exception that proves the rule," Oncogene, vol. 25, no. 38, pp. 5233-5243, 2006.

[93] R. Di Fiore, A. D’Anneo, G. Tesoriere, and R. Vento, "RB1 in cancer: different mechanisms of RB1 inactivation and alterations of pRb pathway in tumorigenesis," Journal of Cellular Physiology, vol. 228, no. 8, pp. 1676-1687, 2013.
[94] J. H. Roelfsema and D. J. M. Peters, "Rubinstein-Taybi syndrome: clinical and molecular overview," Expert Reviews in Molecular Medicine, vol. 9, no. 23, pp. 1-16, 2007.

[95] M. Le Gallo, A. J. O’Hara, M. L. Rudd et al., "Exome sequencing of serous endometrial tumors identifies recurrent somatic mutations in chromatin-remodeling and ubiquitin ligase complex genes," Nature Genetics, vol. 44, no. 12, pp. 1310-1315, 2012.

[96] A. Djos, S. Fransson, P. Kogner, and T. Martinsson, "Aneuploidy in neuroblastoma tumors is not associated with inactivating point mutations in the STAG2 gene," BMC Medical Genetics, vol. 14, no. 1, article 102, 2013.

[97] C. F. Taylor, F. M. Platt, C. D. Hurst, H. H. Thygesen, and M. A. Knowles, "Frequent inactivating mutations of STAG2 in bladder cancer are associated with low tumour grade and stage and inversely related to chromosomal copy number changes," Human Molecular Genetics, vol. 23, no. 8, pp. 1964-1974, 2014.

[98] M. S. Hussain, S. M. Baig, S. Neumann et al., "CDK6 associates with the centrosome during mitosis and is mutated in a large pakistani family with primary microcephaly," Human Molecular Genetics, vol. 22, no. 25, pp. 5199-5214, 2013.

[99] J. M. Davison, M. Yee, J. M. Krill-Burger et al., “The degree of segmental aneuploidy measured by total copy number abnormalities predicts survival and recurrence in superficial gastroesophageal adenocarcinoma," PLoS ONE, vol. 9, no. 1, Article ID e79079, 2014.

[100] R. Loganantharaj and J. Chung, "Integrating diverse information to gain more insight into microarray analysis," Journal of Biomedicine and Biotechnology, vol. 2009, Article ID 648987, 10 pages, 2009. 

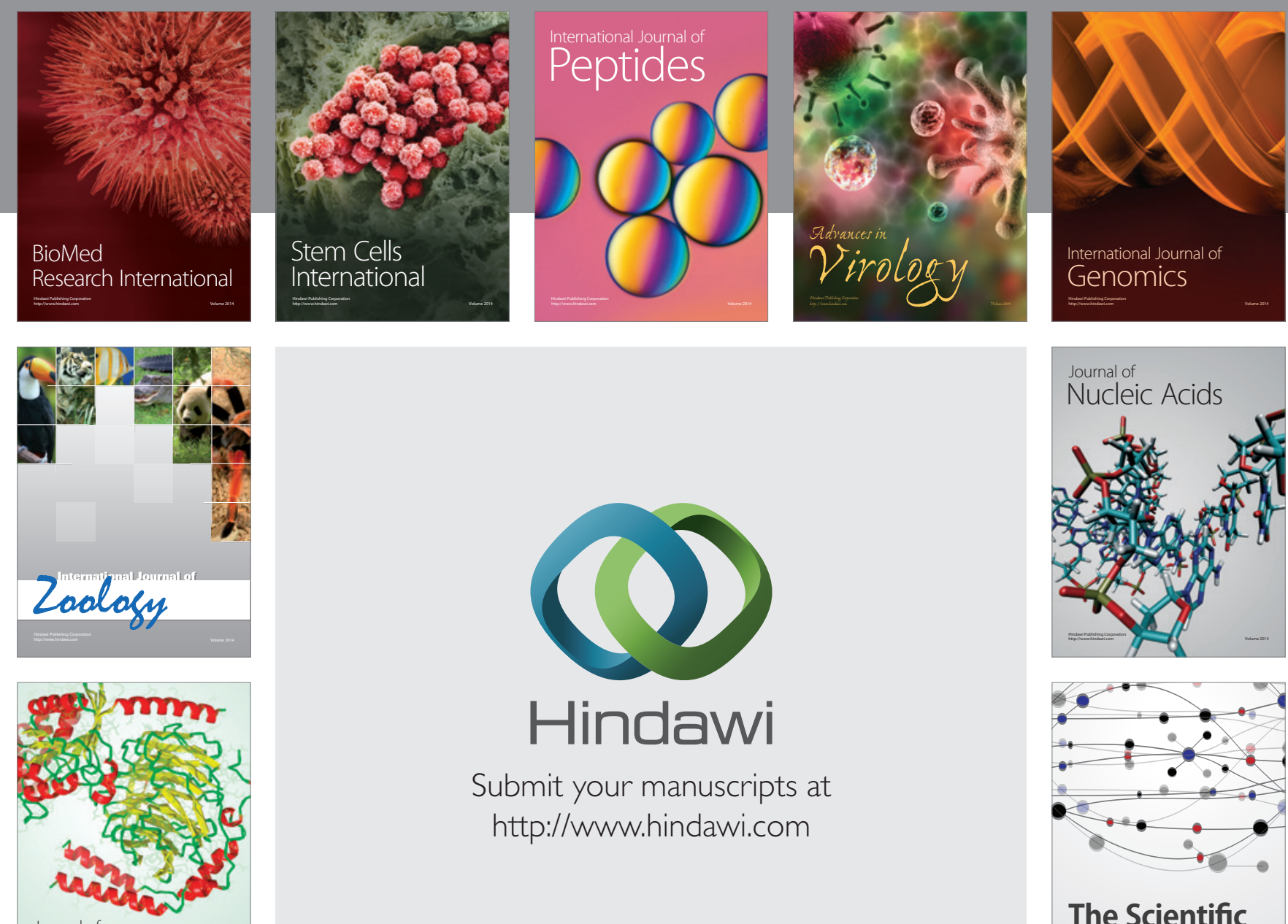

Submit your manuscripts at

http://www.hindawi.com

Journal of
Signal Transduction
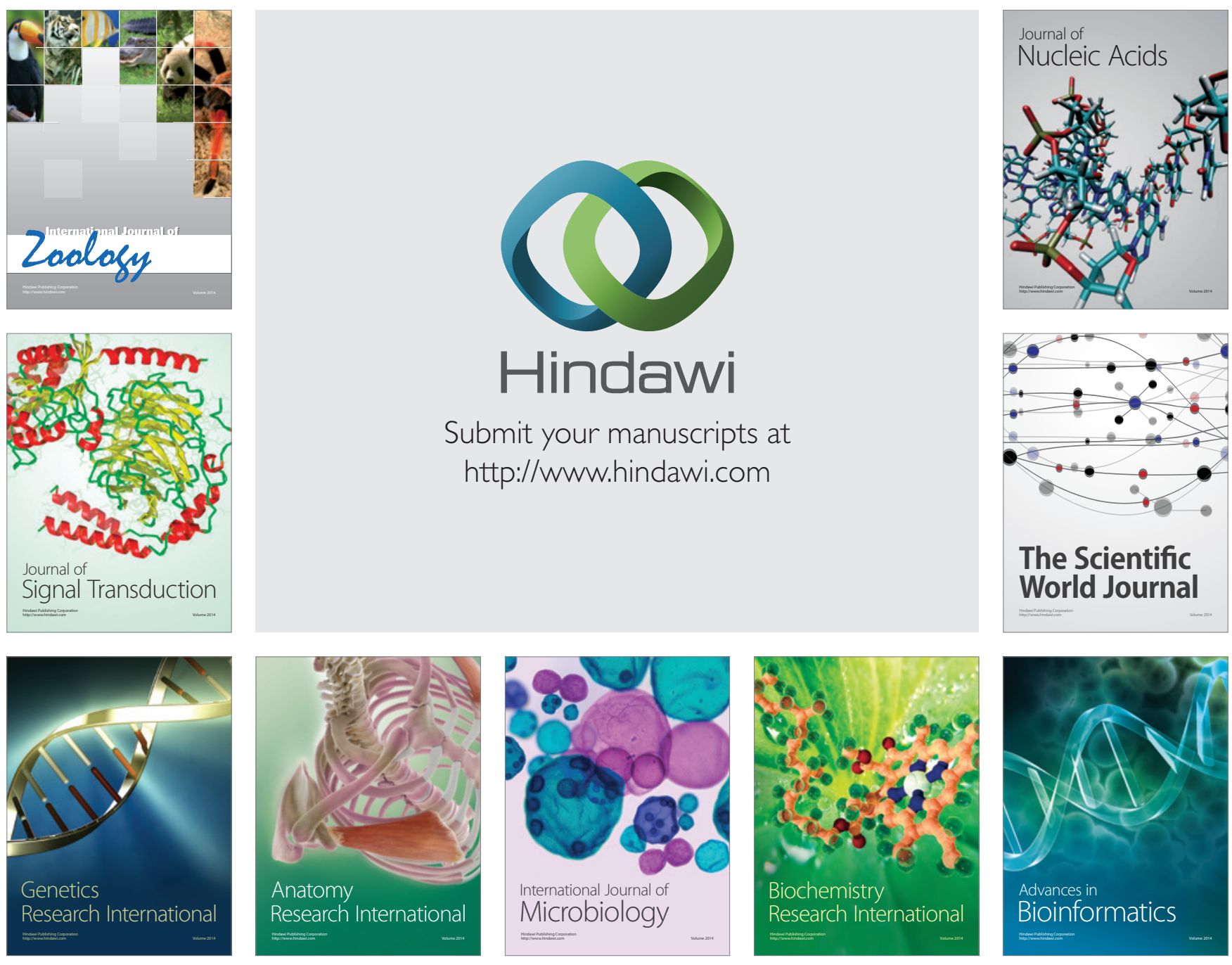

The Scientific World Journal
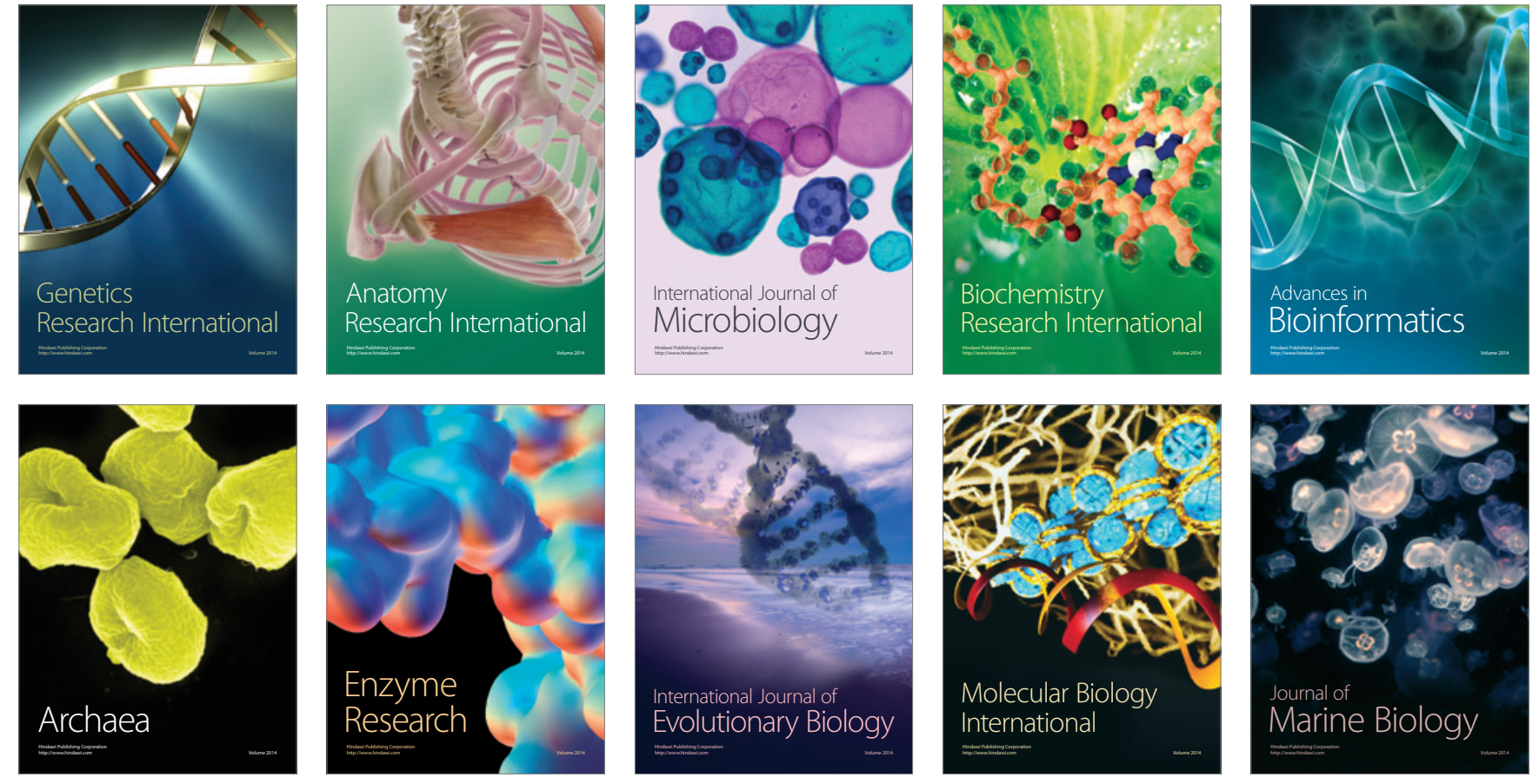